TRANSACTIONS OF THE

AMERICAN MATHEMATICAL SOCIETY

Volume 362, Number 5, May 2010, Pages 2619-2639

S 0002-9947(09)05001-6

Article electronically published on December 14, 2009

\title{
POSITIVE POLYNOMIALS AND SEQUENTIAL CLOSURES OF QUADRATIC MODULES
}

\author{
TIM NETZER
}

\begin{abstract}
Let $\mathcal{S}=\left\{x \in \mathbb{R}^{n} \mid f_{1}(x) \geq 0, \ldots, f_{\mathcal{s}}(x) \geq 0\right\}$ be a basic closed semi-algebraic set in $\mathbb{R}^{n}$ and let $\mathrm{PO}\left(f_{1}, \ldots, f_{s}\right)$ be the corresponding preordering in $\mathbb{R}\left[X_{1}, \ldots, X_{n}\right]$. We examine for which polynomials $f$ there exist identities

$$
f+\varepsilon q \in \mathrm{PO}\left(f_{1}, \ldots, f_{s}\right) \text { for all } \varepsilon>0 .
$$

These are precisely the elements of the sequential closure of $\mathrm{PO}\left(f_{1}, \ldots, f_{s}\right)$ with respect to the finest locally convex topology. We solve the open problem from Kuhlmann, Marshall, and Schwartz (2002, 2005), whether this equals the double dual cone

$$
\mathrm{PO}\left(f_{1}, \ldots, f_{s}\right)^{\vee \vee}
$$

by providing a counterexample. We then prove a theorem that allows us to obtain identities for polynomials as above, by looking at a family of fibrepreorderings, constructed from bounded polynomials. These fibre-preorderings are easier to deal with than the original preordering in general. For a large class of examples we are thus able to show that either every polynomial $f$ that is nonnegative on $\mathcal{S}$ admits such representations, or at least the polynomials from $\operatorname{PO}\left(f_{1}, \ldots, f_{s}\right)^{\vee \vee}$ do. The results also hold in the more general setup of arbitrary commutative algebras and quadratic modules instead of preorderings.
\end{abstract}

\section{INTRODUCTION}

Finitely many real polynomials $f_{1}, \ldots, f_{s} \in \mathbb{R}[\underline{X}]=\mathbb{R}\left[X_{1}, \ldots, X_{n}\right]$ define a basic closed semi-algebraic set

$$
\mathcal{S}=\mathcal{S}\left(f_{1}, \ldots, f_{s}\right)=\left\{x \in \mathbb{R}^{n} \mid f_{1}(x) \geq 0, \ldots, f_{s}(x) \geq 0\right\} .
$$

One is interested in finding characterizations of $\operatorname{Pos}(\mathcal{S})$, the set of all polynomials that are nonnegative on $\mathcal{S}$. Obvious candidates for such nonnegative polynomials are the elements of

$$
\operatorname{PO}\left(f_{1}, \ldots, f_{s}\right):=\left\{\sum_{e \in\{0,1\}^{s}} \sigma_{e} f_{1}^{e_{1}} \cdots f_{s}^{e_{s}} \mid \sigma_{e} \in \sum \mathbb{R}[\underline{X}]^{2}\right\},
$$

the so-called preordering generated by $f_{1}, \ldots, f_{s}$. Many works in Real Algebra and Real Algebraic Geometry deal with the question how $\operatorname{PO}\left(f_{1}, \ldots, f_{s}\right)$ relates to $\operatorname{Pos}(\mathcal{S})$. In dimension one, equality occurs often, at least if the $f_{i}$ are the socalled natural generators for $\mathcal{S}$ (see [KM, KMS]). In dimension two, equality is a

Received by the editors July 21, 2008.

2000 Mathematics Subject Classification. Primary 44A60, 14P10, 13J30; Secondary 11E25.

Key words and phrases. Moment problems, semi-algebraic sets, real algebra, positive polynomials and sum of squares.

(C)2009 American Mathematical Society Reverts to public domain 28 years from publication 
much more uncommon phenomenon. For example, not every globally nonnegative polynomial in two variables is a sum of squares of polynomials. However, Scheiderer has given two local global principles that yield equality between $\mathrm{PO}\left(f_{1}, \ldots, f_{s}\right)$ and $\operatorname{Pos}(\mathcal{S})$ under certain conditions, in the case that $\mathcal{S}$ is compact and two-dimensional (see [Sc2, $\underline{\mathrm{Sc} 3}$ ] and also [M1, $\mathrm{CKM}]$ ). The results require a good behavior of the curves bounding $\mathcal{S}$. Noncompact two-dimensional examples where equality holds are even more rare. One of the few known examples is the preordering generated by $1-X^{2}$ in $\mathbb{R}[X, Y]$; see $\mathrm{M} 2$. From dimension three upwards, equality between $\mathrm{PO}\left(f_{1}, \ldots, f_{s}\right)$ and $\operatorname{Pos}(\mathcal{S})$ is never possible. This is one of the main results from Sc1.

Of course one can ask questions beside equality. For example, Schmüdgen's famous result from S1] says that in case $\mathcal{S}$ is compact, $\mathrm{PO}\left(f_{1}, \ldots, f_{s}\right)$ contains every polynomial which is strictly positive on $\mathcal{S}$, no matter what generators $f_{1}, \ldots, f_{s}$ of $\mathcal{S}$ are chosen, and independent of the dimension of $\mathcal{S}$. However, this result fails in general if $\mathcal{S}$ is not compact. If $\mathcal{S}$ is very large, for example if it contains a full dimensional cone, then the preordering is indeed far from containing every nonnegative or positive polynomial (see for example [KM, KMS, [N2, PoSc, [Sc4]).

Another question arising in this context concerns the $\mathcal{S}$-Moment Problem. One wants to find a characterization of the linear functionals $L: \mathbb{R}[\underline{X]} \rightarrow \mathbb{R}$ that are integration on $\mathcal{S}$. Haviland's Theorem $([\mathrm{H}]$, see also [M1] provides a necessary and sufficient condition. Namely, $L$ is integration on $\mathcal{S}$ if and only if $L$ is $\geq 0$ on $\operatorname{Pos}(\mathcal{S})$. As a characterization of $\operatorname{Pos}(\mathcal{S})$ is not very simple either, one wants to weaken the condition on $L$ and still apply Haviland's Theorem. More precisely, one wants to know whether $L \geq 0$ on $\mathrm{PO}\left(f_{1}, \ldots, f_{s}\right)$ is sufficient for $L$ to be integration. In view of Haviland's Theorem, that means to ask whether

$$
\operatorname{PO}\left(f_{1}, \ldots, f_{s}\right)^{\vee \vee}=\operatorname{Pos}(\mathcal{S})
$$

holds, where $\mathrm{PO}\left(f_{1}, \ldots, f_{s}\right)^{\vee}$ denotes the double dual cone of the preordering. One says that the preordering has the Strong Moment Property (SMP) in this case. For example, if $\mathcal{S}$ is compact, then by the above-mentioned Schmüdgen Theorem, the preordering always has (SMP). There are also many noncompact examples. S2 gives a criterion to decide this question, involving fibre-preorderings constructed from bounded polynomials.

Now in $\mathrm{KM}$, KMS, the following preordering is considered:

$$
\mathrm{PO}\left(f_{1}, \ldots, f_{s}\right)^{\ddagger}:=\left\{f \in \mathbb{R}[\underline{X}] \mid \exists q \in \mathbb{R}[\underline{X}] \forall \varepsilon>0 f+\varepsilon q \in \mathrm{PO}\left(f_{1}, \ldots, f_{s}\right)\right\} .
$$

This definition does not use linear functionals and is therefore better accessible to algebraic methods. $\mathrm{PO}\left(f_{1}, \ldots, f_{s}\right)^{\ddagger}$ turns out to be the sequential closure of $\mathrm{PO}\left(f_{1}, \ldots, f_{s}\right)$ with respect to the finest locally convex topology on $\mathbb{R}[\underline{X}]$, whereas $\mathrm{PO}\left(f_{1}, \ldots, f_{s}\right)^{\vee \vee}$ is the closure. The following relations hold:

$$
\mathrm{PO}\left(f_{1}, \ldots, f_{s}\right) \subseteq \mathrm{PO}\left(f_{1}, \ldots, f_{s}\right)^{\ddagger} \subseteq \mathrm{PO}\left(f_{1}, \ldots, f_{s}\right)^{\vee \vee} \subseteq \operatorname{Pos}(\mathcal{S}) .
$$

In $[\mathrm{Sw1}$, Theorem 5.1, it is shown that every element from $\operatorname{Pos}(\mathcal{S})$ belongs to the sequential closure of the preordering in a certain localization of the polynomial ring. A slightly weaker version of that is [M1, Theorem 6.2.3.

The authors of [KM, KMS, $\mathrm{Po}$ prove $\mathrm{PO}\left(f_{1}, \ldots, f_{s}\right)^{\ddagger}=\operatorname{Pos}(\mathcal{S})$ (and therefore (SMP)) for certain classes of preorderings in the polynomial ring. The question whether in polynomial rings

$$
\mathrm{PO}\left(f_{1}, \ldots, f_{s}\right)^{\ddagger}=\mathrm{PO}\left(f_{1}, \ldots, f_{s}\right)^{\vee \vee}
$$


always holds remained open in these works (see Open Problem 3 in $[\mathrm{KM}$ and Open Problem 2 in $[\mathrm{KMS}]$ ). We solve this problem by providing a counterexample; $\mathrm{PO}\left(f_{1}, \ldots, f_{s}\right)^{\ddagger}$ does not equal $\mathrm{PO}\left(f_{1}, \ldots, f_{s}\right)^{\vee \vee}$ in general.

Then it is natural to ask for conditions under which equality holds. It is also interesting to ask whether a fibre theorem in the spirit of [S2] can be established for $\mathrm{PO}\left(f_{1}, \ldots, f_{s}\right)^{\ddagger}$ instead of $\mathrm{PO}\left(f_{1}, \ldots, f_{s}\right)^{\vee \vee}$. This question already turned up in S2. Our counterexample implies that such a general theorem will require stricter assumptions than Schmüdgen's Fibre Theorem.

Theorem 5.3 from [KMS] is such a fibre theorem for finitely generated preorderings that describe cylinders with compact cross section. We will generalize this result to quadratic modules in arbitrary commutative algebras.

We will then deduce criteria for

$$
\mathrm{PO}\left(f_{1}, \ldots, f_{s}\right)^{\ddagger}=\mathrm{PO}\left(f_{1}, \ldots, f_{s}\right)^{\vee \vee}
$$

and

$$
\mathrm{PO}\left(f_{1}, \ldots, f_{s}\right)^{\ddagger}=\operatorname{Pos}(\mathcal{S})
$$

to hold. They allow applications that go beyond the known examples from [KM, KMS, $\mathrm{Po}$.

\section{Notation AND PRELIMINARIES}

For the following results on topological vector spaces we refer to $[\mathrm{B}$, Chapter II. Let $E$ be an $\mathbb{R}$-vector space. The finest locally convex topology on $E$ is the vector space topology having the collection of all convex, absorbing and symmetric subsets of $E$ as a neighborhood base of zero. All the linear functionals on $E$ are continuous, $E$ is Hausdorff, and every finite dimensional subspace of $E$ inherits the canonical topology. By [Sf], Chapter 2, Exercise 7(b), a sequence in $E$ converges if and only if it lies in a finite dimensional subspace of $E$ and converges there. So for the sequential closure of a set $M$ in $E$, defined as the set of all limits of sequences from $M$, and denoted by $M^{\ddagger}$, we have

$$
M^{\ddagger}=\bigcup_{W} \overline{M \cap W},
$$

where the union runs over all finite dimensional subspaces $W$ of $E$. From now on, we will restrict ourselves to convex cones in $E$, i.e. to subsets $M$ that are closed under addition and multiplication with positive reals. The following alternative characterization for $M^{\ddagger}$ is included from [CMN2]:

Proposition 2.1. Let $M$ be a convex cone in $E$ and let $f \in E$. The following are equivalent:

(1) $f$ is the limit of a sequence of elements of $M$.

(2) $\exists q \in E$ such that $f+\epsilon q \in M$ for each real $\epsilon>0$.

Proof. (2) $\Rightarrow(1)$ is clear. (1) $\Rightarrow(2)$ : Let $f=\lim _{i \rightarrow \infty} f_{i}, f_{i} \in M$. As mentioned above, the subspace of $V$ spanned by $f_{1}, f_{2}, \ldots$ is finite dimensional. Let $w_{1}, \ldots, w_{N} \in M$ be a basis for this subspace. Then $f_{i}=\sum_{j=1}^{N} r_{i j} w_{j}, f=$ $\sum_{j=1}^{N} r_{j} w_{j}$, with $r_{i j}, r_{i} \in \mathbb{R}, r_{j}=\lim _{i \rightarrow \infty} r_{i j}$. Let $q:=\sum_{j=1}^{N} w_{j}$. Then, for any real $\epsilon>0, r_{i j}<r_{j}+\epsilon$ for $i$ sufficiently large, so $f+\epsilon q=\sum_{j=1}^{N}\left(r_{j}+\epsilon\right) w_{j}=$ $\sum_{j=1}^{N} r_{i j} w_{j}+\sum_{j=1}^{N}\left(r_{j}+\epsilon-r_{i j}\right) w_{j}=f_{i}+\sum_{j=1}^{N}\left(r_{j}+\epsilon-r_{i j}\right) w_{j} \in M$. 
So the proposition gives the alternative description

$$
M^{\ddagger}=\{f \in E \mid \exists q \in E \forall \varepsilon>0 f+\varepsilon q \in M\} .
$$

For preorderings $M$ in $\mathbb{R}$-algebras, the object $M^{\ddagger}$ was first introduced in $[\mathrm{KM}]$ in terms of this last characterization. It only turned out later to be the sequential closure of $M$.

The closure of a convex cone $M$ is denoted by $\bar{M}$. [B], Chapter II.39, Corollary 5 implies $\bar{M}=M^{\vee \vee}$ for convex cones. Here, $M^{\vee \vee}$ denotes the double dual cone of $M$, i.e. the set

$$
\left\{x \in E \mid L(x) \geq 0 \text { for all } L: E \rightarrow \mathbb{R} \text { linear with } L(M) \subseteq \mathbb{R}_{\geq 0}\right\} .
$$

We obviously have $M \subseteq M^{\ddagger} \subseteq M^{\vee \vee}$. If $E$ has countable vector space dimension, then a set $M$ in $E$ is closed if and only if its intersection with every finite dimensional subspace of $E$ is closed, by [Bi], Proposition 1. So $M$ is closed if and only if it is sequentially closed, i.e. if $M=M^{\ddagger}$ holds. This implies that the (transfinite) sequence of iterated sequential closures of $M$ terminates at $\bar{M}$ in that case. Note that in case $E$ is not countable dimensional, then this sequence may terminate before it reaches the closure. It indeed always terminates at the closure with respect to the topology of finitely open sets, which can be smaller in the case of uncountable dimension. We refer to CMN1 for more information.

Now let $A$ be a commutative $\mathbb{R}$-algebra with 1 . Of course $A$ is an $\mathbb{R}$-vector space, and we equip it with the finest locally convex topology. A quadratic module is a set $M \subseteq A$ with $M+M \subseteq M, 1 \in M$ and $A^{2} \cdot M \subseteq M$. Here, $A^{2}$ denotes the set of squares in $A$. A preordering is a quadratic module with the additional property $M \cdot M \subseteq M$. Quadratic modules (and preorderings) are convex cones, and we look at $M^{\ddagger}$ and $M^{\vee \vee}$, defined as above.

For a set $S \subseteq A$, the smallest quadratic module/preordering containing $S$ is called the quadratic module/preordering generated by $S$. We write $\operatorname{QM}(S)$ and $\mathrm{PO}(S)$ for it, respectively. $\mathrm{QM}(S)$ consists of all finite sums of elements $\sigma$ and $\sigma \cdot f$, where $f \in S$ and $\sigma$ is a sum of squares in $A \cdot \operatorname{PO}(S)$ consists of all finite sums of elements $\sigma f_{1} \cdots f_{t}$, where $\sigma$ is a sum of squares, $t \geq 0$ and all $f_{i} \in S$. Of special interest is the case that $S$ is finite. We then call $\mathrm{QM}(S)$ and $\mathrm{PO}(S)$ finitely generated.

An important notion, introduced in $\mathrm{PoSc}$, is that of stability of a finitely generated quadratic module. If $M$ is generated by $S=\left\{a_{1}, \ldots, a_{s}\right\}$, then we call $M$ stable, if for every finite dimensional $\mathbb{R}$-subspace $U$ of $A$ there is another finite dimensional $\mathbb{R}$-subspace $V$ of $A$, such that

$$
M \cap U \subseteq\left\{\sigma_{0}+\sigma_{1} a_{1}+\cdots+\sigma_{s} a_{s} \mid \sigma_{i} \in \sum V^{2}\right\} .
$$

A map that assigns to each $U$ such a $V$ is then called a stability map. Whereas the notion of stability is independent of the choice of generators of $M$, the stability map is not. We refer the reader to [N2, $\mathrm{PoSc}, \mathrm{Sc} 4]$ for proofs and details.

To $A$ there corresponds the set of all real characters on $A$, i.e.

$$
\mathcal{V}_{A}:=\{\alpha: A \rightarrow \mathbb{R} \mid \alpha \text { a unital } \mathbb{R} \text {-algebra homomorphism }\} .
$$

Elements $a$ from $A$ define functions $\hat{a}$ on $\mathcal{V}_{A}$ by $\hat{a}(\alpha):=\alpha(a)$. We equip $\mathcal{V}_{A}$ with the coarsest topology making all these functions continuous. As the functions $\hat{a}$ separate points, this makes $\mathcal{V}_{A}$ a Hausdorff space, and we have the algebra homomorphism

$$
\text { ^: } A \rightarrow C\left(\mathcal{V}_{A}, \mathbb{R}\right) \text {. }
$$


If $A$ is finitely generated as an $\mathbb{R}$-algebra, then $\mathcal{V}_{A}$ embeds into some $\mathbb{R}^{n}$, by taking generators $x_{1}, \ldots, x_{n}$ and sending $\alpha$ to $\left(\alpha\left(x_{1}\right), \ldots, \alpha\left(x_{n}\right)\right)$. So $\mathcal{V}_{A}$ is the zero set in $\mathbb{R}^{n}$ of the kernel of the algebra homomorphism $\pi: \mathbb{R}\left[X_{1}, \ldots, X_{n}\right] \rightarrow A ; X_{i} \mapsto x_{i}$. The use of an element $a$ from $A$ as a function $\hat{a}$ coincides with the usual use of $a$ as a polynomial function on that embedded variety. In particular, the topology on $\mathcal{V}_{A}$ is inherited from the canonical one on $\mathbb{R}^{n}$. Note also that $A$ is a countable dimensional $\mathbb{R}$-vector space in case it is finitely generated as an $\mathbb{R}$-algebra.

Now we again consider arbitrary commutative $\mathbb{R}$-algebras $A$ with 1 . For a set $M \subseteq A$, without loss of generality a quadratic module, we define

$$
\mathcal{S}(M)=\left\{\alpha \in \mathcal{V}_{A} \mid \alpha(M) \subseteq \mathbb{R}_{\geq 0}\right\} .
$$

If $M$ is finitely generated, then $\mathcal{S}(M)$ is called a basic closed semi-algebraic set. For any set $Y \subseteq \mathcal{V}_{A}$ we define

$$
\operatorname{Pos}(Y):=\{a \in A \mid \hat{a} \geq 0 \text { on } Y\} .
$$

This is a preordering. Starting with a quadratic module or a preordering $M$ in $A$, we have the following chain:

$$
M \subseteq M^{\ddagger} \subseteq M^{\vee \vee} \subseteq \operatorname{Pos}(\mathcal{S}(M)) .
$$

The last inclusion comes from the fact that characters on $A$ are in particular linear functionals. As for example proven in [CMN1, $M^{\ddagger}$ and $M^{\vee \vee}$ are again quadratic modules, even preorderings if $M$ was a preordering. Following [KM], KMS, S2], we make the following definitions.

Definition 2.2. $\quad$ (i) $M$ has the strong moment property (SMP) if $M^{\vee \vee}=$ $\operatorname{Pos}(\mathcal{S}(M))$ holds.

(ii) $M$ has the $\ddagger$-property if $M^{\ddagger}=\operatorname{Pos}(\mathcal{S}(M))$ holds.

(iii) $M$ is saturated if $M=\operatorname{Pos}(\mathcal{S}(M))$ holds.

(iv) $M$ is closed if $M=M^{\vee \vee}$ holds.

The interest in $M^{\vee \vee}$ and (SMP) is motivated by a classical theorem by Haviland. For certain classes of algebras, it states that a linear functional on $A$ is integration with respect to some measure on $\mathcal{S}(M)$ if and only if it is nonnegative on $\operatorname{Pos}(\mathcal{S}(M)$ ) ([H] in the case that $A$ is a polynomial algebra; see [M1] for a more general version). So if $M$ has (SMP), then every functional that is nonnegative on $M$ is integration on $\mathcal{S}(M)$. Nonnegativity on $M$ is a priori a much weaker condition than nonnegativity on $\operatorname{Pos}(\mathcal{S}(M))$. This is one of the reasons that make (SMP) so interesting.

A method to decide whether (SMP) holds for a finitely generated preordering $P$ in $\mathbb{R}[\underline{X}]$ is given in $[\underline{S 2}$, as mentioned in the introduction. Let $b$ be a polynomial that is bounded on $\mathcal{S}(P)$. Then

$$
P^{\vee \vee}=\bigcap_{r \in \mathbb{R}}(P+(b-r))^{\vee \vee}
$$

holds, where $(b-r)$ denotes the ideal generated by $b-r$. This implies that $P$ has (SMP) if and only if all the preorderings $P+(b-r)$ have (SMP). As these so-called fibre preorderings usually describe lower dimensional semi-algebraic sets, they are easier to deal with in general. The result in particular implies that every finitely generated preordering in $\mathbb{R}[\underline{X}]$ describing a compact set $\mathcal{S}(P)$ has (SMP). This was already part of the earlier paper [S1]. For an alternative proof of the result from [S2], see also [M1, N1]. 
The ‡-property was introduced and studied in [KM, KMS] for polynomial algebras. The authors for example show that in case $\mathcal{S}(P)$ is a cylinder with compact cross section, then the preordering $P$ has the $\ddagger$-property, under reasonable assumptions on the generators of $P$. This is also shown, using different methods, in $[\mathrm{Po}$.

It was an open problem in $\mathrm{KM}$, KMS, whether (SMP) and the $\ddagger$-property are equivalent or even whether $M^{\ddagger}=M^{\vee \vee}$ is always true. We start by showing that the answer to both questions is negative.

\section{A counterexample}

The example in this section will answer Open Problem 3 in $[\mathrm{KM}$ and Open Problem 2 in $\mathrm{KMS}$ in the negative. It will also give a negative answer to the question in S2, whether the fibre theorem holds for the $\ddagger$-property instead of (SMP).

Consider $A=\mathbb{R}[X, Y]$, the polynomial algebra in two variables, and $f_{1}=$ $Y^{3}, f_{2}=Y+X, f_{3}=1-X Y$ and $f_{4}=1-X^{2} \in A$. These polynomials define the following basic closed semi-algebraic set $\mathcal{S}\left(f_{1}, \ldots, f_{4}\right)$ in $\mathcal{V}_{A}=\mathbb{R}^{2}$ :

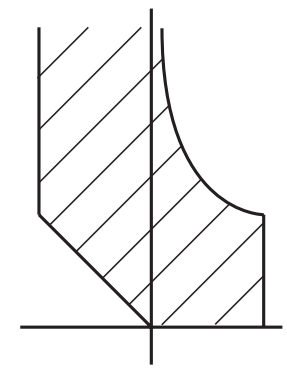

Proposition 3.1. The preordering $P=\mathrm{PO}\left(f_{1}, f_{2}, f_{3}, f_{4}\right)$ in $\mathbb{R}[X, Y]$ has (SMP).

Proof. The polynomial $X$ is bounded on $\mathcal{S}\left(f_{1}, \ldots, f_{4}\right)$, and we can apply Schmüdgen's fibre theorem from [S2 to $P$. For any $r \in \mathbb{R} \backslash[-1,0]$, the preordering

$$
P_{r}:=P+(X-r)=\operatorname{PO}\left(f_{1}, \ldots, f_{4}, X-r, r-X\right)
$$

describes a compact semi-algebraic set and therefore has (SMP) by [S1] (even the ( $\ddagger$-property). For $r \in[-1,0]$, the preordering $\mathrm{PO}\left(Y^{3}, Y+r, 1-r Y\right) \subseteq \mathbb{R}[Y]$ is saturated. This follows from $[\mathrm{KM}$, Theorem 2.2. So

$$
P_{r}=\mathrm{PO}\left(f_{1}, \ldots, f_{4}, X-r, r-X\right)
$$

in $\mathbb{R}[X, Y]$ is saturated as well. In particular, $P_{r}$ has (SMP). So by [S2], the whole preordering $P$ has (SMP).

The next result is a characterization of $P^{\ddagger}$. We write

$$
\mathrm{PO}\left(a_{1}, \ldots, a_{s}\right)_{d}
$$

for the set of elements having a representation in $\mathrm{PO}\left(a_{1}, \ldots, a_{s}\right)$ with sums of squares of elements of degree $\leq d$.

Proposition 3.2. A polynomial $f \in \mathbb{R}[X, Y]$ belongs to $\mathrm{PO}\left(f_{1}, f_{2}, f_{3}, f_{4}\right)^{\ddagger}$ if and only if there is some $d \in \mathbb{N}$ such that for all $r \in[-1,1], f(r, Y)$ belongs to $\mathrm{PO}\left(f_{1}(r, Y), \ldots, f_{4}(r, Y)\right)_{d}$ in $\mathbb{R}[Y]$. 
Proof. The "if"-part is a consequence of Theorem 4.3 below (or can already be obtained by looking at the proof of Theorem 5.3 in [KMS]).

For the "only if"-part assume $f$ belongs to $\operatorname{PO}\left(f_{1}, \ldots, f_{4}\right)^{\ddagger}$. So there is some $q \in \mathbb{R}[X, Y]$ and sums of squares $\sigma_{e}^{(\varepsilon)} \in \sum \mathbb{R}[X, Y]$ for all $\varepsilon>0$ and $e \in\{0,1\}^{4}$ such that

$$
f+\varepsilon q=\sum_{e} \sigma_{e}^{(\varepsilon)} f_{1}^{e_{1}} \cdots f_{4}^{e_{4}} .
$$

Note that the total degree of the $\sigma_{e}^{(\varepsilon)}$ may rise with $\varepsilon$ getting smaller. However, the degree as polynomials in $Y$ cannot rise; it is bounded by the $Y$-degree of $f+\varepsilon q$, which does not change with $\varepsilon$. This is because the set $\mathcal{S}\left(f_{1}, \ldots, f_{4}\right)$ contains the cylinder $[-1,0] \times[1, \infty]$, so whenever one adds two polynomials which are nonnegative on it, the leading terms as polynomials in $Y$ cannot cancel. So the degree in $Y$ of the sum is the maximum of the $Y$-degrees of the terms.

By evaluating in $X=r$, this means that $f(r, Y)+\varepsilon q(r, Y)$ belongs to

$$
\mathrm{PO}\left(f_{1}(r, Y), \ldots, f_{4}(r, Y)\right)_{d}
$$

for some fixed $d$ and all $r \in[-1,1], \varepsilon>0$. But by [PoSc], Proposition 2.6, this is a closed set in a finite dimensional subspace of $\mathbb{R}[Y]$. So we get $f(r, Y) \in$ $\mathrm{PO}\left(f_{1}(r, Y), \ldots, f_{4}(r, Y)\right)_{d}$ for all $r \in[-1,1]$, the desired result.

Corollary 3.3. The preordering $P=\mathrm{PO}\left(f_{1}, \ldots, f_{4}\right)$ does not have the $\ddagger$-property.

Proof. The polynomial $Y$ is obviously nonnegative on $\mathcal{S}\left(f_{1}, \ldots, f_{4}\right)$. However, it does not belong to the preordering

$$
\mathrm{PO}\left(f_{1}(1, Y), \ldots, f_{4}(1, Y)\right)=\mathrm{PO}\left(Y^{3}, Y+1,1-Y\right) \subseteq \mathbb{R}[Y]
$$

Indeed, writing down a representation and evaluating in $Y=0$, this shows that $Y^{2}$ divides $Y$, a contradiction. So in view of Proposition 3.2, $Y$ cannot belong to $P^{\ddagger}$.

Remark 3.4. Note that $Y$ is not in $P^{\ddagger}$ as it fails to be in the preordering corresponding to the fibre $X=1$. However, Proposition 3.2 even demands all the polynomials $f(r, Y)$ to have representations in the fibre-preorderings

$$
\mathrm{PO}\left(f_{1}(r, Y), \ldots, f_{4}(r, Y)\right)
$$

with simultaneous degree bounds, for $f$ to be in $P^{\ddagger}$. Indeed, there are examples of polynomials belonging to all of the fibre-preorderings, but failing the degree-bound condition (and so also not belonging to $P^{\ddagger}$ ). We will give one here, as it gives a justification for one of the assumptions in Theorems 4.1 and 4.3 below.

Example 3.5. Take $f=2 Y+X$, which belongs to $\operatorname{Pos}\left(\mathcal{S}\left(f_{1}, \ldots, f_{4}\right)\right)$. For any $r \in[-1,1], f(r, Y)=2 Y+r$ belongs to $\mathrm{PO}\left(f_{1}(r, Y), \ldots, f_{4}(r, Y)\right)$ : for $r>0$ as $f(r, Y)$ is strictly positive on the corresponding compact semi-algebraic set (so use [S1]); for $r \in[-1,0]$, the fibre preordering is saturated, as mentioned in the proof of Proposition 3.1

However, for $r \searrow 0$, there can be no bound on the degree of the sums of squares in the representation. Indeed, for $r>0$, write down a representation

$$
2 Y+r=\sum_{e \in\{0,1\}^{3}} \sigma_{e}^{(r)} Y^{3 e_{1}}(Y+r)^{e_{2}}(1-r Y)^{e_{3}},
$$


where the $\sigma_{e}^{(r)}$ are sums of squares. Evaluating in $Y=0$, this shows

$$
\sigma_{(0,1,0)}^{(r)}(0)+\sigma_{(0,1,1)}^{(r)}(0) \leq 1 .
$$

Now if the degrees of the $\sigma_{e}^{(r)}$ could be bounded for all $r>0$, we could write down a first-order logic formula saying that we have representations as in (11) for all $r>0$. We add the statement (2) to the formula. By Tarski's Transfer Principle, it holds in any real closed extension field of $\mathbb{R}$. So take such a representation in some non-Archimedean real closed extension field $R$ for some $r>0$ which is infinitesimal with respect to $\mathbb{R}$. The same argument, as for example in [KMS, Example 4.4 (a), shows that we can apply the residue map $\mathcal{O} \rightarrow \mathcal{O} / \mathfrak{m}=\mathbb{R}$ to the coefficients of all the polynomials occurring in this representation. Here, $\mathcal{O}$ denotes the convex hull of $\mathbb{R}$ in $R$. This is a valuation ring with maximal ideal $\mathfrak{m}$.

So we get a representation

$$
\begin{aligned}
2 Y= & \sigma_{(0,0,0)}+\sigma_{(1,0,0)} Y^{3}+\sigma_{(0,1,0)} Y+\sigma_{(0,0,1)}+\sigma_{(1,1,0)} Y^{4} \\
& +\sigma_{(1,0,1)} Y^{3}+\sigma_{(0,1,1)} Y+\sigma_{(1,1,1)} Y^{4}
\end{aligned}
$$

with sums of squares $\sigma_{e}$ in $\mathbb{R}[Y]$ fulfilling

$$
\sigma_{(0,1,0)}(0)+\sigma_{(0,1,1)}(0) \leq 1 .
$$

As no cancellation of highest degree terms can occur, we get

$$
0=\sigma_{(0,0,0)}=\sigma_{(1,0,0)}=\sigma_{(0,0,1)}=\sigma_{(1,1,0)}=\sigma_{(1,0,1)}=\sigma_{(1,1,1)}
$$

as well as

$$
\sigma_{(0,1,0)}+\sigma_{(0,1,1)}=2 .
$$

This last fact obviously contradicts (3).

So for $2 Y+X$, the degree bound condition on the fibres fails, although the polynomial belongs to all of the fibre preorderings. In view of Proposition [3.2, it does not belong to $\mathrm{PO}\left(f_{1}, \ldots, f_{4}\right)^{\ddagger}$. This shows that the "degree bound" assumption in Theorems 4.1 and 4.3 below is really necessary.

Note also that the example is an explicit illustration of Theorem 8.2.6 from [PD, where the general impossibility of a certain degree bound for Schmüdgen's Theorem from [S1] is proved.

Remark 3.6. The above example answers the question in $\underline{\mathbf{S 2}}$, whether the fibre theorem holds for the $\ddagger$-property instead of (SMP). Indeed, we have shown in the proof of Proposition 3.1 that all the fibre preorderings $P_{r}$ do not only have (SMP), but even the $\ddagger$-property. As $P$ itself does not have the $\ddagger$-property, this gives a negative answer to the question. However, we will prove a result below that allows us to use a dimension reduction when examining the $\$$-property under certain conditions.

\section{A FIBRE THEOREM FOR SEQUENTIAL CLOSURES}

We want to prove a fibre theorem in the spirit of [S2], to be able to examine the sequential closure of a quadratic module in terms of (easier) fibre-modules. We consider the following general setup. Let $A, B$ be commutative $\mathbb{R}$-algebras with 1 , let $X$ be a compact Hausdorff space, and assume we have algebra homomorphisms 
$\varphi: B \rightarrow A$ and ${ }^{\wedge}: B \rightarrow C(X, \mathbb{R})$ (homomorphisms are always assumed to map 1 to $1)$ :

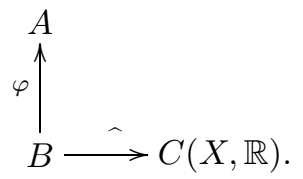

We assume that the image of $B$ in $C(X, \mathbb{R})$ separates points of $X$, i.e. for any two distinct points $x, y \in X$ there is some $b \in B$ such that $\hat{b}(x) \neq \hat{b}(y)$. The StoneWeierstrass Theorem then implies that $\widehat{B}$ is dense in $C(X, \mathbb{R})$ with respect to the sup-norm.

For $x \in X$ let $I_{x}:=\{b \in B \mid \hat{b}(x)=0\}$ be the vanishing ideal of $x$ in $B$, and $J_{x}$ the ideal in $A$ generated by $\varphi\left(I_{x}\right)$. The homomorphism $\varphi$ makes $A$ a $B$-module in the usual sense of commutative algebra (not to be confused with quadratic modules!). For a $B$-submodule $W$ of $A$ we write

$$
J_{x}(W)=\left\{\sum_{i=1}^{t} w_{i} \varphi\left(b_{i}\right) \mid t \in \mathbb{N}, w_{i} \in W, b_{i} \in I_{x}\right\} .
$$

So $J_{x}(W)$ is a $B$-submodule of $W$. We have $J_{x}(A)=J_{x}$.

Now let $M \subseteq A$ be a quadratic module. For any $x \in X, M+J_{x}$ is again a quadratic module, called the fibre-module of $M$ with respect to $x$. Our first goal is to prove the following abstract fibre theorem:

Theorem 4.1. Let $A, B$ be commutative $\mathbb{R}$-algebras, $X$ a compact Hausdorff space, $\varphi: B \rightarrow A$ and $\hat{\imath}: B \rightarrow C(X, \mathbb{R})$ algebra homomorphisms, such that $\hat{B}$ separates points of $X$. Let $M \subseteq A$ be a quadratic module and assume $\varphi(b) \in M$ whenever $\hat{b}>0$ on $X$. Then for any finitely generated $B$-submodule $W$ of $A$ we have

$$
\bigcap_{x \in X} M+J_{x}(W) \subseteq M^{\ddagger} .
$$

The requirement on $W$ can be understood as a "degree bound condition" as in Proposition 3.2. Example 3.5 shows that $\bigcap_{x \in X} M+J_{x} \subseteq M^{\ddagger}$ is not true under the remaining assumptions in general (we will discuss this in more detail below).

To prove the theorem, we first need the following technical proposition.

Proposition 4.2. Let $A, B$ be $\mathbb{R}$-algebras and $\varphi: B \rightarrow A$ an algebra homomorphism. Let $X$ be a compact Hausdorff space and ${ }^{\wedge} B \rightarrow C(X, \mathbb{R})$ an algebra homomorphism whose image separates points of $X$. Assume $a, a_{1}, \ldots, a_{l} \in A$ and $\varepsilon>0$ are such that for all $x \in X$ there is an identity

$$
a=\sum_{i=1}^{l} \varphi\left(b_{i}^{(x)}\right) \cdot a_{i},
$$

with $b_{i}^{(x)} \in B$ and $\left|\widehat{b_{i}^{(x)}}(x)\right|<\varepsilon$ for all $i$. Then there are $b_{1}, \ldots, b_{l} \in B$ with $\left|\hat{b}_{i}\right|<\varepsilon$ on $X$ for all $i$ and

$$
a=\sum_{i=1}^{l} \varphi\left(b_{i}\right) \cdot a_{i} .
$$


Proof. Every $x \in X$ has an open neighborhood $U_{x}$, such that $\left|\widehat{b_{i}^{(x)}}\right|<\varepsilon$ on $U_{x}$ for all $i=1, \ldots, l$. By compactness of $X$ there are $x_{1}, \ldots, x_{t} \in X$, such that

$$
X=U_{x_{1}} \cup \cdots \cup U_{x_{t}} .
$$

If $t=1$, then the result follows, so assume $t \geq 2$. Choose a partition of unity $e_{1}, \ldots, e_{t}$ subordinate to that cover, i.e. all $e_{k}$ are continuous functions from $X$ to $[0,1], \operatorname{supp}\left(e_{k}\right) \subseteq U_{x_{k}}$ for all $k$, and $e_{1}(x)+\cdots+e_{t}(x)=1$ for all $x \in X$. Then for

$$
f_{i}:=e_{1} \cdot \widehat{b_{i}^{\left(x_{1}\right)}}+\cdots+e_{t} \cdot \widehat{b_{i}^{\left(x_{t}\right)}}
$$

we have

$$
\left\|f_{i}\right\|<\varepsilon
$$

where \|\| denotes the sup-norm on $C(X, \mathbb{R})$. Let

$$
\delta:=\min \left\{\varepsilon-\left\|f_{i}\right\| \mid i=1, \ldots, l\right\}
$$

and choose a positive real number $N$, large enough to bound the sup-norm of all $\widehat{b_{i}^{\left(x_{k}\right)}}$.

The image of $B$ in $C(X, \mathbb{R})$ is dense, by the Stone-Weierstrass Theorem. So we find $q_{1}, \ldots, q_{t-1} \in B$ such that

$$
\left\|e_{k}-\hat{q}_{k}\right\|<\frac{\delta}{N(t-1) t}
$$

for $k=1, \ldots, t-1$, and we define

$$
q_{t}:=1-\sum_{k=1}^{t-1} q_{k} .
$$

So we have for $k=1, \ldots, t$,

$$
\left\|e_{k}-\hat{q}_{k}\right\|<\frac{\delta}{N t} .
$$

We define

$$
b_{i}:=q_{1} \cdot b_{i}^{\left(x_{1}\right)}+\cdots+q_{t} \cdot b_{i}^{\left(x_{t}\right)}
$$

for $i=1, \ldots, l$. So

$$
\begin{aligned}
\left\|\hat{b}_{i}\right\| & \leq\left\|f_{i}\right\|+\left\|\hat{b}_{i}-f_{i}\right\| \\
& \leq\left\|f_{i}\right\|+\sum_{k=1}^{t}\left\|e_{k}-\hat{q}_{k}\right\| \cdot\left\|\widehat{b_{i}^{\left(x_{k}\right)}}\right\| \\
& <\left\|f_{i}\right\|+\delta \\
& \leq \varepsilon
\end{aligned}
$$


Now as $\sum_{k=1}^{t} q_{k}=1$ we have

$$
\begin{aligned}
a & =\varphi\left(\sum_{k=1}^{t} q_{k}\right) \cdot a \\
& =\sum_{k=1}^{t}\left(\varphi\left(q_{k}\right) \cdot \sum_{i=1}^{l} \varphi\left(b_{i}^{\left(x_{k}\right)}\right) a_{i}\right) \\
& =\sum_{i=1}^{l} \varphi\left(\sum_{k=1}^{t} q_{k} b_{i}^{\left(x_{k}\right)}\right) \cdot a_{i} \\
& =\sum_{i=1}^{l} \varphi\left(b_{i}\right) \cdot a_{i},
\end{aligned}
$$

which proves the proposition.

Now we can give the proof of Theorem 4.1. It is a generalization of the proof of Theorem 5.3 from [KMS.

Proof of Theorem 4.1. Fix a finitely generated $B$-submodule $W$ of $A$. Assume $f \in$ $A$ has a representation

$$
f=m_{x}+j_{x}
$$

with $m_{x} \in M$ and $j_{x} \in J_{x}(W)$, for all $x \in X$. As $J_{x}(W) \subseteq W$, we can assume without loss of generality that $m_{x} \in M \cap W$ for all $x$. Let $a_{1}, \ldots, a_{l}$ be generators of $W$ as a $B$-module. Due to the identity $a=\left(\frac{a+1}{2}\right)^{2}-\left(\frac{a-1}{2}\right)^{2}$ we can assume that all $a_{j}$ are squares in $A$ (by possibly enlarging $W$ ). We will now show that

$$
f+\varepsilon \sum_{i=1}^{l} a_{i} \in M
$$

for all $\varepsilon>0$. Therefore fix one such $\varepsilon>0$. We take representations

$$
j_{x}=\sum_{i=1}^{l} \varphi\left(c_{i}^{(x)}\right) \cdot a_{i}, \quad m_{x}=\sum_{i=1}^{l} \varphi\left(d_{i}^{(x)}\right) \cdot a_{i},
$$

where all $c_{i}^{(x)}, d_{i}^{(x)} \in B$ and $\widehat{c_{i}^{(x)}}(x)=0$. Now each $x \in X$ has an open neighborhood $U_{x}$, such that

$$
\left|\widehat{c_{i}^{(x)}}\right|<\frac{\varepsilon}{2} \text { on } U_{x}
$$

for $i=1, \ldots, l$. By compactness of $X$ we have

$$
X=U_{x_{1}} \cup \cdots \cup U_{x_{t}}
$$

for some $x_{1}, \ldots, x_{t} \in X$. Let $e_{1}, \ldots, e_{t}$ be a continuous partition of unity subordinate to that cover. Using the Stone-Weierstrass Theorem, we approximate the square root of each $e_{k}$ (which is again a continuous function) by elements $g_{k}$ from $B$, such that

$$
\sum_{k=1}^{t}\left\|e_{k}-\hat{g}_{k}^{2}\right\| \cdot\left\|\widehat{d_{i}^{\left(x_{k}\right)}}\right\|<\frac{\varepsilon}{2}
$$


holds for all $i=1, \ldots, l$. Here, \|\| denotes the sup-norm on $C(X, \mathbb{R})$ again. Define

$$
a=f-\underbrace{\sum_{k=1}^{t} \varphi\left(g_{k}\right)^{2} \cdot m_{x_{k}}}_{\in M} .
$$

The proof is complete if we show that $a+\varepsilon \sum_{i=1}^{l} a_{i} \in M$. Fix $x \in X$. Then

$$
\begin{aligned}
a= & \sum_{k=1}^{t} e_{k}(x) \cdot f-\sum_{k=1}^{t} \varphi\left(g_{k}^{2}\right) \cdot m_{x_{k}} \\
= & \sum_{k=1}^{t} e_{k}(x) \cdot(\underbrace{f-m_{x_{k}}}_{=j_{x_{k}}})+\sum_{k=1}^{t}\left(e_{k}(x)-\varphi\left(g_{k}^{2}\right)\right) m_{x_{k}} \\
= & \sum_{k=1}^{t} e_{k}(x) \sum_{i=1}^{l} \varphi\left(c_{i}^{\left(x_{k}\right)}\right) a_{i}+\sum_{k=1}^{t}\left(e_{k}(x)-\varphi\left(g_{k}^{2}\right)\right) \sum_{i=1}^{l} \varphi\left(d_{i}^{\left(x_{k}\right)}\right) a_{i} \\
= & \sum_{i=1}^{l}\left(\sum_{k=1}^{t} e_{k}(x) \varphi\left(c_{i}^{\left(x_{k}\right)}\right)\right) \cdot a_{i} \\
& \quad+\sum_{i=1}^{l}\left(\sum_{k=1}^{t}\left(e_{k}(x)-\varphi\left(g_{k}^{2}\right)\right) \varphi\left(d_{i}^{\left(x_{k}\right)}\right)\right) \cdot a_{i} \\
= & \sum_{i=1}^{l} \varphi\left(b_{i}^{(x)}\right) \cdot a_{i},
\end{aligned}
$$

where we define

$$
b_{i}^{(x)}=\sum_{k=1}^{t} e_{k}(x) \cdot c_{i}^{\left(x_{k}\right)}+\left(e_{k}(x)-g_{k}^{2}\right) \cdot d_{i}^{\left(x_{k}\right)} .
$$

By the above considerations we have

$$
\left|\widehat{b_{i}^{(x)}}(x)\right|<\varepsilon
$$

for all $i$. So we can apply Proposition 4.2 to $a, a_{1}, \ldots, a_{l}$ and find

$$
a=\sum_{i=1}^{l} \varphi\left(b_{i}\right) \cdot a_{i}
$$

for some $b_{i} \in B$ with $\left|\hat{b}_{i}\right|<\varepsilon$ on $X$. Thus

$$
a+\varepsilon \sum_{i=1}^{l} a_{i}=\sum_{i=1}^{l} \varphi\left(b_{i}+\varepsilon\right) \cdot a_{i} \in M,
$$

as all $\widehat{b_{i}+\varepsilon}$ are strictly positive on $X$ and all $a_{i}$ are squares.

We demonstrate how to apply Theorem 4.1 for a given algebra $A$ and a quadratic module $M \subseteq A$. Therefore assume there are $b_{1}, \ldots, b_{t} \in A$ with $\Lambda_{i}-b_{i}, b_{i}-\lambda_{i} \in M$ for real numbers $\lambda_{i} \leq \Lambda_{i}(i=1, \ldots, t)$. This of course implies that each $b_{i}$ is bounded as a function on $\mathcal{S}(M) \subseteq \mathcal{V}_{A}$, but the converse is not always true. Let $B=\mathbb{R}\left[b_{1}, \ldots, b_{t}\right]$ be the subalgebra of $A$ generated by the $b_{i}$ and let $\varphi: B \rightarrow A$ 
be the canonical inclusion. Let $\widetilde{M}$ be the quadratic module in $B$ generated by $\Lambda_{1}-b_{1}, b_{1}-\lambda_{1}, \ldots, \Lambda_{t}-b_{t}, b_{t}-\lambda_{t}$. It is Archimedean, for example by [JP, Theorem 4.1. Let $X=\mathcal{S}(\widetilde{M}) \subseteq \mathcal{V}_{B}$, so $X$ is compact, and the canonical homomorphism : $B \rightarrow C(X, \mathbb{R})$ fulfills the separating points condition. Now whenever some $\hat{b}$ is strictly positive on $X$, then $b \in \widetilde{M}$, by [J], Theorem 6 (see also [PD], Theorem 5.3.6 and [M1, Theorem 5.4.4). So $\varphi(b) \in M$. For any $x \in X$, we have $\hat{b}_{i}(x) \in\left[\lambda_{i}, \Lambda_{i}\right]$, and

$$
J_{x}(W)=\left\{\sum_{i=1}^{t}\left(b_{i}-\hat{b}_{i}(x)\right) w_{i} \mid w_{i} \in W\right\}
$$

holds for any $B$-module $W$. Thus write for $r=\left(r_{1}, \ldots, r_{t}\right) \in \Lambda=\prod_{i=1}^{t}\left[\lambda_{i}, \Lambda_{i}\right]$,

$$
J_{r}(W)=\left\{\sum_{i=1}^{t}\left(b_{i}-r_{i}\right) w_{i} \mid w_{i} \in W\right\}
$$

and $J_{r}:=J_{r}(A)=\left(b_{1}-r_{1}, \ldots, b_{t}-r_{t}\right)$. If $M$ is finitely generated as a quadratic module, then $M+J_{r}$ is also finitely generated, by the generators of $M$ and $\pm\left(b_{1}-r_{1}\right), \ldots, \pm\left(b_{t}-r_{t}\right)$. The following fibre theorem is our main result.

Theorem 4.3. Let $A$ be a commutative $\mathbb{R}$-algebra and $M \subseteq A$ a quadratic module. Suppose $b_{1}, \ldots, b_{t} \in A$ are such that

$$
\Lambda_{1}-b_{1}, b_{1}-\lambda_{1}, \ldots, \Lambda_{t}-b_{t}, b_{t}-\lambda_{t} \in M
$$

for some real numbers $\lambda_{i} \leq \Lambda_{i}(i=1, \ldots, t)$. Then for every finitely generated $\mathbb{R}\left[b_{1}, \ldots, b_{t}\right]$-submodule $W$ of $A$ we have

$$
\bigcap_{r \in \Lambda} M+J_{r}(W) \subseteq M^{\ddagger},
$$

where $\Lambda=\prod_{i=1}^{t}\left[\lambda_{i}, \Lambda_{i}\right]$. In particular, if $M$ is finitely generated and all the (finitely generated) quadratic modules $M+J_{r}$ are closed and stable with the same stability map, then $M^{\ddagger}=\bar{M}$. If all $M+J_{r}$ are saturated and stable with the same stability map, then $M$ has the $\ddagger$-property. (Here, the stability map with respect to the canonical generators of each $M+J_{r}$ is meant.)

Proof. The first part of the theorem is clear from the above considerations and Theorem 4.1. For the second part, assume $M$ is finitely generated, say by $f_{1}, \ldots, f_{s}$. Then $M+J_{r}$ is finitely generated as a quadratic module, by the canonical generators

$$
f_{1}, \ldots, f_{s}, \pm\left(b_{1}-r_{1}\right), \ldots, \pm\left(b_{t}-r_{t}\right) .
$$

Assume all $M+J_{r}$ are closed (or saturated, respectively) and stable with the same stability map. Suppose some $f$ belongs to $\bar{M}$ (or $\operatorname{Pos}(\mathcal{S}(M))$, respectively). Then $f$ belongs to all $\overline{M+J_{r}}$ (or $\operatorname{Pos}\left(\mathcal{S}\left(M+J_{r}\right)\right)$, respectively), so to all $M+J_{r}$ by our assumption. Now by the assumed stability there is a fixed finite dimensional $\mathbb{R}$-subspace $W$ of $A$, such that $f$ belongs to all $M+J_{r}(W)$. So the first part of the theorem yields $f \in M^{\ddagger}$.

Remark 4.4. (1) In Example 3.5 the polynomial $f=2 Y+X$ belongs to all the preorderings $\mathrm{PO}\left(f_{1}, \ldots, f_{4}\right)+(X-r)$ in $A=\mathbb{R}[X, Y]$. However, there is no finitely generated $\mathbb{R}[X]$-submodule $W$ of $\mathbb{R}[X, Y]$ such that $f$ belongs to all $\mathrm{PO}\left(f_{1}, \ldots, f_{4}\right)+$ $J_{r}(W)$. This follows from what we have shown in Example 3.5. We have also seen that $f$ does not belong to $\mathrm{PO}\left(f_{1}, \ldots, f_{4}\right)^{\ddagger}$. Note that $X+1,1-X$ in $\mathrm{PO}\left(f_{1}, \ldots, f_{4}\right)$ 
is fulfilled, as $f_{4}=1-X^{2}$, and using an easy calculation as for example in [KM], Note 2.3 (4). So the "degree bound condition" is necessary in Theorem 4.3 and also in Theorem 4.1 .

(2) Example 5.6 below will show that the assumption $\Lambda_{i}-b_{i}, b_{i}-\lambda_{i} \in M$ for all $i$ cannot be omitted in Theorem 4.3. So the same is true for the assumption

$$
\hat{b}>0 \text { on } X \Rightarrow \varphi(b) \in M
$$

in Theorem 4.1

(3) In the case that all the occurring quadratic fibre-modules $M+J_{r}$ in Theorem 4.3 are saturated and stable with the same stability map, we get a little bit more than the $\ddagger$-property for $M$. We obtain that for every finite dimensional subspace $V$ of $A$ there is some $q_{V} \in A$ such that whenever $f \in \operatorname{Pos}(\mathcal{S}(M)) \cap V$, then $f+\varepsilon q_{V} \in M$ for all $\varepsilon>0$. In other words, the polynomial $q$ from the $\ddagger$-property does only depend on the subspace $f$ is taken from, not on the explicit choice of $f$. This follows from the proof of Theorem 4.1, noting that $q$ does only depend on the $B$-module $W$, which depends only on $V$ and the stability map here.

\section{Applications and examples}

In this section we give some applications of the fibre theorem. The first one is the Cylinder Theorem (Theorem 5.3 combined with Corollary 5.5) from [KMS. See [KM], KMS] for the definition of natural generators for semi-algebraic subsets of $\mathbb{R}$.

Corollary 5.1. Let $P=\mathrm{PO}\left(f_{1}, \ldots, f_{s}\right)$ be a finitely generated preordering in the polynomial ring $\mathbb{R}\left[X_{1}, \ldots, X_{n}, Y\right]$. Assume $N-\sum_{i=1}^{n} X_{i}^{2} \in P$ for some $N>0$. Now for all $r \in \mathbb{R}^{n}$, the preordering

$$
\mathrm{PO}\left(f_{1}(r, Y), \ldots, f_{s}(r, Y)\right) \subseteq \mathbb{R}[Y]
$$

describes a basic closed semi-algebraic set $S_{r}$ in $\mathbb{R}$. Suppose the natural generators for $S_{r}$ are among the $f_{1}(r, Y), \ldots, f_{s}(r, Y)$, whenever $S_{r}$ is not empty. Then $P$ has the $\ddagger$-property.

If all the fibre sets $S_{r}$ are of the form $\emptyset,(-\infty, \infty),(-\infty, p],[q, \infty),(-\infty, p] \cup[q, \infty)$ or $[p, q]$, then the result holds with $P$ replaced by $M=\mathrm{QM}\left(f_{1}, \ldots, f_{s}\right)$.

Proof. The assumptions imply that all the preorderings

$$
P+\left(X_{1}-r_{1}, \ldots, X_{n}-r_{n}\right)
$$

(or the corresponding quadratic modules, respectively) are saturated and stable with the same stability map for all $r$. See [KMS], Section 4. An easy calculation, as for example in [KM], Note $2.3(4)$, shows that

$$
\sqrt{N}-X_{i}, X_{i}+\sqrt{N} \in P
$$

for all $i$. So we can apply Theorem 4.3 ,

We can also use Theorem 4.3 in the case that the natural generators are not among the $f_{i}(r, Y)$. This can be seen as a generalization of Corollary 5.4 from KMS:

Corollary 5.2. Let $M=\operatorname{QM}\left(f_{1}, \ldots, f_{s}\right)$ be a finitely generated quadratic module in $\mathbb{R}\left[X_{1}, \ldots, X_{n}, Y\right]$ and assume $N-\sum_{i=1}^{n} X_{i}^{2} \in M$ for some $N>0$. Suppose for all 
$r \in \mathbb{R}^{n}$ that the set $S_{r}$ (defined as in Corollary [5.1) is either empty or unbounded. Then

$$
M^{\ddagger}=\bar{M}
$$

holds.

Proof. Again $\sqrt{N}-X_{i}, X_{i}+\sqrt{N} \in P$ for all $i$. Furthermore, the assumptions imply that all the quadratic modules

$$
M+\left(X_{1}-r_{1}, \ldots, X_{n}-r_{n}\right)
$$

are closed and stable with the same stability map for all $r$ (for the empty fibers use Theorem 4.5 from [KMS ]. Now apply Theorem 4.3 .

We want to get results for more complicated fibres. [Sc2] gives a criterion for quadratic modules on curves to be stable and closed. However, we need some result to obtain the uniform stability asked for in Theorem 4.3. So we consider the following setup. Let $b \in \mathbb{R}[X, Y]$ be a polynomial of degree $d>0$. We assume that the highest degree homogeneous part of $b$ factors as

$$
\prod_{i=1}^{d}\left(r_{i} X+s_{i} Y\right),
$$

where all the $\left(r_{i}: s_{i}\right)$ are pairwise disjoint points of $\mathbb{P}^{1}(\mathbb{R})$. In particular, $b$ is square free. Let $C$ denote the affine curve in $\mathbb{A}^{2}$ defined by $b$ and $\widetilde{C}$ its projective closure in $\mathbb{P}^{2}$. So $\widetilde{C}$ is defined by $\tilde{b}$, the homogenization of $b$ with respect to the new variable $Z$. The assumption on the highest degree part of $b$ implies that all the points at infinity of $b$, namely

$$
P_{1}=\left(-s_{1}: r_{1}: 0\right), \ldots, P_{d}=\left(-s_{d}: r_{d}: 0\right) \in \mathbb{P}^{2},
$$

are real regular points (of the projective curve $\widetilde{C}$ ). So the local rings of $\widetilde{C}$ at all these points are discrete valuation rings (a well-known fact; see for example $[\mathrm{F}$, Chapter 3 ). Indeed, the projective curve $\widetilde{C}$ is the so-called "good completion" (see for example [ $\mathrm{Pl}]$ ) of the affine curve $C$. We denote the valuation corresponding to the local ring at $P_{i}$ by $\operatorname{ord}_{P_{i}}$. For a polynomial $h \in \mathbb{R}[X, Y]$, we write $\operatorname{ord}_{P_{i}}(h)$ and mean the value with respect to the valuation $\operatorname{ord}_{P_{i}}$ of $h\left(\frac{X}{Z}, \frac{Y}{Z}\right)$ as a rational function on $\tilde{C}$.

We start with the following result:

Proposition 5.3. Let $b, C$ and $\widetilde{C}$ be as above. Suppose

$$
\operatorname{ord}_{P_{i}}(h) \geq-l
$$

for some $h \in \mathbb{R}[X, Y], l \in \mathbb{N}$ and all $i$. Then there is some $h^{\prime} \in \mathbb{R}[X, Y]$ with $\operatorname{deg}\left(h^{\prime}\right) \leq l$ and $h \equiv h^{\prime} \bmod (b)$.

Proof. Let $m$ be the degree of $h$ and $\tilde{b}=Z^{d} b\left(\frac{X}{Z}, \frac{Y}{Z}\right)$ as well as $\tilde{h}=Z^{m} h\left(\frac{X}{Z}, \frac{Y}{Z}\right)$ be the homogenization of $b$ and $h$, respectively. Assume, without loss of generality,

$$
P_{1}=(1: y: 0)
$$

for some $y \in \mathbb{R}$. 
For any homogeneous polynomial $g$ in the variables $X, Y, Z$ we have

$$
0 \leq \operatorname{ord}_{P_{1}}\left(\frac{g}{X^{\operatorname{deg}(g)}}\right)=I\left(P_{1} ; \tilde{b} \cap g\right),
$$

where $I$ denotes the intersection number. This is $[\mathrm{F}]$, Chapter 3.3.

As

$$
\operatorname{ord}_{P_{1}}(h)=\operatorname{ord}_{P_{1}}\left(\frac{\tilde{h}}{X^{m}}\right)-m \cdot \operatorname{ord}_{P_{1}}\left(\frac{Z}{X}\right),
$$

we have

$$
\begin{aligned}
-l & \leq \operatorname{ord}_{P_{1}}(h) \\
& =I\left(P_{1} ; \tilde{b} \cap \tilde{h}\right)-m \cdot I\left(P_{1} ; \tilde{b} \cap Z\right) \\
& \leq I\left(P_{1} ; \tilde{b} \cap \tilde{h}\right)-m .
\end{aligned}
$$

Now whenever $m \geq l+1$, then

$$
1 \leq I\left(P_{1} ; \tilde{b} \cap \tilde{h}\right)
$$

so $\tilde{h}$ must vanish at $P_{1}$.

The same argument applies to all points at infinity of $b$. So if $m \geq l+1$, then the highest degree part of $b$ divides the highest degree part of $h$ in $\mathbb{R}[X, Y]$. Thus $h$ can be reduced modulo $b$ to a polynomial $h^{\prime}$ of strictly smaller degree.

In the following proposition, the pure closedness and stability result follows from Sc2, Proposition 6.5.

Proposition 5.4. Let $M=\mathrm{QM}\left(f_{1}, \ldots, f_{s}\right) \subseteq \mathbb{R}[X, Y]$ be a finitely generated quadratic module. Let $b \in \mathbb{R}[X, Y]$ be a polynomial whose highest degree part factors as above. For some $r \in \mathbb{R}$ assume that all the points at infinity of the curve $C_{r}$ defined by $b=r$ lie in the closure of $\mathcal{S}(M) \cap C_{r}(\mathbb{R})$. Then the finitely generated quadratic module

$$
M+(b-r)=\mathrm{QM}\left(f_{1}, \ldots, f_{s}, b-r, r-b\right)
$$

is closed and stable, with a stability map that depends only on $b$ and $f_{1}, \ldots, f_{s}$, but not on $r$.

Proof. Without loss of generality, let $P_{1}=(1: y: 0)$ be a point at infinity of $C_{r}$. Denote by $\operatorname{ord}_{P_{1}}$ the valuation with respect to the local ring of $\widetilde{C_{r}}$ at $P_{1}$. Let $h \in \mathbb{R}[X, Y]$ have degree $m$, and let $\tilde{h}$ as well as $\widetilde{b-r}$ be the homogenizations, as in the previous proof. Then

$$
\begin{aligned}
\operatorname{ord}_{P_{1}}(h) & =\operatorname{ord}_{P_{1}}\left(\frac{\tilde{h}}{X^{m}}\right)-m \cdot \operatorname{ord}_{P_{1}}\left(\frac{Z}{X}\right) \\
& \geq-m \cdot I\left(P_{1} ; \widetilde{b-r} \cap Z\right) \\
& =-m \cdot I\left(P_{1} ; \tilde{b} \cap Z\right),
\end{aligned}
$$

where the last equality uses property $(7)$ in $[\mathrm{F}$, p. 75 , for intersection numbers. So there is some $N$, not depending on $r$, such that

$$
\operatorname{ord}_{P}(h) \geq-m \cdot N
$$

for all the points of infinity of $C_{r}$. 
Now the proof of Proposition 6.5 from [Sc3] shows that whenever $h \in \overline{M+(b-r)}$, then we can find a representation

$$
h=\sum_{i=0}^{s} \sigma_{i} f_{i}+g \cdot(b-r)
$$

with sums of squares $\sigma_{i}$ built of polynomials that have order greater than $-m \cdot N$ in all points at infinity of $C_{r}$. Applying Proposition 5.3 we can reduce these elements modulo $b-r$ and obtain a representation as in (4) with sums of squares of elements of degree less than or equal to $m \cdot N$. So of course the degree of $g$ is also bounded suitably, independent of $r$. This shows that the stability map does not depend on $r$.

So the following theorem is an immediate consequence of Theorem 4.3 and Proposition 5.4

Theorem 5.5. Let $M \subseteq \mathbb{R}[X, Y]$ be a finitely generated quadratic module. Let $b \in \mathbb{R}[X, Y]$ with $\Lambda-b, b-\lambda \in M$ for some $\lambda \leq \Lambda$, and assume the highest degree part of $b$ factors as above. Suppose that for all $r \in[\lambda, \Lambda]$, all the points at infinity of the curve $C_{r}$ defined by $b=r$ lie in the closure of $\mathcal{S}(M) \cap C_{r}(\mathbb{R})$. Then

$$
M^{\ddagger}=\bar{M}
$$

holds. If all the fibre modules $M+(b-r)$ have (SMP) in addition, then $M$ has the †-property.

We give some explicit examples for these last results.

Example 5.6. We look at the semi-algebraic set in $\mathbb{R}^{2}$ defined by the inequalities

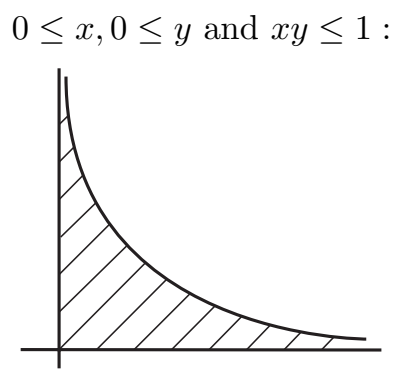

A lot of interesting phenomena can be observed for this set. There are different quadratic modules describing it. We consider the following ones:

$$
\begin{aligned}
M_{1} & :=\mathrm{QM}(X, Y, 1-X Y), \\
M_{2} & :=\mathrm{QM}(X, Y, X Y, 1-X Y), \\
M_{3} & :=\mathrm{QM}\left(X, Y^{3}, X Y, 1-X Y\right), \\
P & :=\operatorname{PO}(X, Y, 1-X Y) .
\end{aligned}
$$

The quadratic module $M_{1}$ is stable; one checks that no cancellation of highest degree terms can occur in a sum

$$
\sigma_{0}+\sigma_{1} X+\sigma_{2} Y+\sigma_{3}(1-X Y)
$$

So by [Sc4], Theorem 5.4, $M_{1}$ does not have (SMP), and by PoSc], Proposition 2.6 , it is closed. 
To the quadratic module $M_{2}$ we can apply Theorem 5.5 with the polynomial $b=X Y$ : we have $b, 1-b \in M_{2}$. For $r \in[0,1]$, the finitely generated quadratic module

$$
\mathrm{QM}(X, Y, X Y, 1-X Y)+(X Y-r)=\mathrm{QM}(X, Y)+(X Y-r)
$$

is saturated. This is an easy calculation for $r>0$; for $r=0$ it is Example 3.26 from [Pl]. So $M_{2}$ has the $\ddagger$-property, and in particular (SMP).

Note that the fibre modules of $M_{1}$ and $M_{2}$ are the same:

$$
M_{1}+(X Y-r)=M_{2}+(X Y-r)
$$

for all $r \in[0,1]$. As $M_{1}$ does not have the $\ddagger$-property, this shows that the condition $\Lambda-b, b-\lambda \in M$ in Theorem 5.5, as well as the corresponding conditions in Theorems 4.3 and 4.1 , cannot be omitted.

Now consider $M_{3}$. The quadratic module $\mathrm{QM}\left(Y^{3}\right) \subseteq \mathbb{R}[Y]$, obtained by factoring out the ideal $(X)$, does not have (SMP) (see for example $[\mathrm{KM}]$ ). So in view of Proposition 4.8 from [Sc4, $M_{3}$ also does not have (SMP). On the other hand, we can still apply Theorem 5.5 with $b=X Y$ and obtain

$$
M_{3}^{\ddagger}=\overline{M_{3}} .
$$

Last, the preordering $P$ obviously contains $M_{2}$ and therefore also has the $\ddagger-$ property. This solves the question posed in [KMS, Example 8.4.

Example 5.7. We consider the semi-algebraic set defined by the inequalities

$$
0 \leq x(x+y)(x-y)-x y \leq 1:
$$

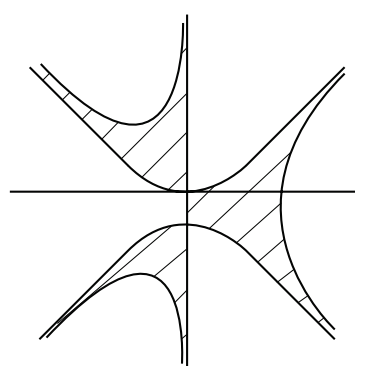

We can apply Theorem 5.5 to the quadratic module

$$
M=\mathrm{QM}(b, 1-b),
$$

where $b=X(X+Y)(X-Y)-X Y$. We use $b$ as the bounded polynomial and obtain

$$
M^{\ddagger}=\bar{M} .
$$

However, $M$ does not have (SMP). Indeed, the quadratic module

$$
M+(b)
$$

does not have (SMP). This follows from [P], Theorem 3.17 together with [Sc2], Proposition 6.5. So in view of Proposition 4.8 from [Sc4], $M$ does not have (SMP). 


\section{Applichtions to polynomial optimization}

We want to explain how the result of Theorem 4.3, together with the observation from Remark4.4 (3), can be used for constrained polynomial optimization. We take a similar approach to the one in $[\mathrm{L}$; see also [M1, Chapter 10] and [Sw2 for a nice account of the topic. However, our approach does not only apply to compact semialgebraic sets.

Assume $A=\mathbb{R}[\underline{X}]$, so $\mathcal{V}_{A}=\mathbb{R}^{n}$. Consider a finitely generated quadratic module

$$
M=\mathrm{QM}\left(f_{1}, \ldots, f_{s}\right) \subseteq \mathbb{R}[\underline{X}]
$$

with associated semi-algebraic set $\mathcal{S}=\mathcal{S}(M)$, which has the property

$$
f \geq 0 \text { on } \mathcal{S} \Rightarrow \exists q \text { s.t. } f+\varepsilon q \in M \text { for all } \varepsilon>0,
$$

and assume $q$ can be chosen to only depend on the degree of $f$ (in other words: for any nonnegative polynomial of the same degree, the same $q$ works). Without loss of generality, $q$ can be chosen to be from $M$ (see [KM], the note following Proposition 1.3). Note that for example the quadratic modules from Corollary 5.1 and Theorem 5.5 have this strong property. Note also that if a compact semi-algebraic set is described by a preordering, or more generally, by an Archimedean quadratic module $M$, the above condition holds, as every strictly positive polynomial belongs to $M$. So $q=1$ works for every nonnegative polynomial.

Given some $f \in \mathbb{R}[\underline{X}]$, one wants to calculate the infimum of $f$ on $\mathcal{S}$, denoted by $f_{*}$. This is usually a hard problem, but for compact semi-algebraic sets $S$, Lasserre [L provided a sequence of semi-definite programs (which are much easier to solve), whose optimal values converge to $f_{*}$.

Now take the polynomial $q$ from above corresponding to the degree of $f$. It can be obtained explicitly in the case of Theorem 4.1 from the proof. For example, in Corollary 5.1, $q$ can be chosen to be the sum of the elements

$$
\left(\frac{Y^{j}-1}{2}\right)^{2} \text { and }\left(\frac{Y^{j}+1}{2}\right)^{2}
$$

where $j$ runs from 0 to the $Y$-degree of $f$.

For fixed $\varepsilon>0$ and $d \in \mathbb{N}$, we consider

$$
F_{\varepsilon, d}:=\sup \left\{r \mid f-r+\varepsilon q \in M_{d}\right\}
$$

and

$$
F_{\varepsilon}:=\sup \{r \mid f-r+\varepsilon q \in M\} .
$$

Here, $M_{d}$ denotes the set of all elements from $M$ that can be represented by $f_{1}, \ldots, f_{s}$ and sums of squares of degree $\leq d$.

We obviously have $F_{\varepsilon, d} \leq F_{\varepsilon}$ for all $d$. Furthermore, each $F_{\varepsilon, d}$ can be obtained by solving a semi-definite program, which can be done efficiently; see [L, M1, Sw2].

Proposition 6.1. The sequence $\left(F_{\varepsilon, d}\right)_{d \in \mathbb{N}}$ converges monotonically increasing to $F_{\varepsilon}$.

Proof. It is clear that the sequence is monotonically increasing. Now take some $r$ which is feasible for $F_{\varepsilon}$, which means that $f-r+\varepsilon p$ belongs to $M$ (if no such $r$ exists, then the statement is also clear, as all values are $-\infty$ ). But then $f-r+\varepsilon p$ belongs to $M_{d}$ for some large enough $d$. So $F_{\varepsilon, d} \geq r$, which proves the statement. 
Now suppose $f \geq r$ on $\mathcal{S}$ for some $r \in \mathbb{R}$. Then $f-r$ is nonnegative on $\mathcal{S}$ and so

$$
f-r+\varepsilon q \in M
$$

holds for all $\varepsilon>0$. This shows that $F_{\varepsilon} \geq f_{*}$ for all $\varepsilon>0$. We have used here that subtracting $r$ from $f$ does not change the degree, and therefore we can use the polynomial $q$, no matter how large or small $r$ is. This could fail if $M$ has the †-property only, not the stronger version we assume here.

Proposition 6.2. For $\varepsilon \searrow 0$, the sequence $\left(F_{\varepsilon}\right)_{\varepsilon}$ converges monotonically decreasing to $f_{*}$.

Proof. From the fact that $q$ is in $M$ it is clear that the sequence is monotonically decreasing. Now suppose $f_{*}$ is finite and $F_{\varepsilon} \geq f_{*}+\delta$ for some $\delta>0$ and all $\varepsilon>0$. This means that $f_{*}+\frac{\delta}{2}$ is feasible for all $F_{\varepsilon}$, and so

$$
f-f_{*}-\frac{\delta}{2}+\varepsilon q \in M
$$

holds for all $\varepsilon>0$. This clearly implies $f-f_{*}-\frac{\delta}{2} \geq 0$ on $\mathcal{S}$ and so $f \geq f_{*}+\frac{\delta}{2}$ on $\mathcal{S}$, a contradiction.

If $f_{*}=-\infty$, the same argument applies by assuming $F_{\varepsilon} \geq N$ for some $N \in \mathbb{R}$ and all $\varepsilon$.

So combining these results, we get the following:

Theorem 6.3. For every $f \in \mathbb{R}[\underline{X}]$ there is a sequence $\left(m_{i}\right)_{i \in \mathbb{N}}$ of natural numbers, such that the sequence $\left(F_{\frac{1}{i}, m_{i}}\right)_{i}$ converges to $f_{*}$.

\section{ACKNOWLEDGEMENTS}

The author thanks Murray Marshall and Claus Scheiderer for interesting and helpful discussions on the topic of this work.

\section{REFERENCES}

[Bi] T. M. Bisgaard: The Topology of Finitely Open Sets is not a Vector Space Topology, Arch. Math. 60 (1993), 546-552. MR1216700 (94g:46013)

[B] N. Bourbaki, Topological Vector Spaces, Chapters 1-5, English edition, Springer-Verlag, Berlin, 1987. MR910295 (88g:46002)

[CKM] J. Cimprič, S. Kuhlmann, M. Marshall: Positivity in Power Series Rings, Advances in Geometry, to appear.

[CMN1] J. Cimprič, T. Netzer, M. Marshall: On the Real Multidimensional Rational K-Moment Problem, to appear in Trans. Amer. Math. Soc.

[CMN2] J. Cimprič, T. Netzer, M. Marshall: Closures of Quadratic Modules, to appear in Israel J. Math.

[F] W. Fulton: Algebraic Curves, W.A. Benjamin (1969). MR0313252 (47:1807)

[H] E.K. Haviland: On the Momentum Problem for Distribution Functions in more than one Dimension II, Amer. J. Math. 58 (1936), 164-168. MR.1507139

[J] T. Jacobi: A Representation Theorem for Certain Partially Ordered Commutative Rings, Math. Z. 237 (2001), 259-273. MR 1838311(2002e:13048)

[JP] T. Jacobi, A. Prestel: Distinguished Representations of Strictly Positive Polynomials, J. reine angew. Math. 532 (2001), 223-235. MR 1817508 (2001m:14080)

$[\mathrm{KM}] \quad$ S. Kuhlmann, M. Marshall: Positivity, Sums of Squares and the Multi-dimensional Moment Problem, Trans. Amer. Math. Soc. 354 (2002), 4285-4301. MR.1926876 (2003j:14078) 
[KMS] S. Kuhlmann, M. Marshall, N. Schwartz: Positivity, Sums of Squares and the Multi-dimensional Moment Problem II, Adv. Geom. 5 (2005), 583-606. MR2174483 (2006i:14064)

[L] J.B. Lasserre: Global Optimization with Polynomials and the Problem of Moments, SIAM J. Optim. 11 (2001) 796-817. MR.1814045 (2002b:90054)

[M1] M. Marshall: Positive Polynomials and Sums of Squares, AMS Math. Surveys and Monographs 146, Providence (2008). MR2383959 (2009a:13044)

[M2] M. Marshall: Polynomials Non-negative on a Strip, Proc. of the Amer. Math. Soc., to appear.

[N1] T. Netzer: An Elementary Proof of Schmüdgen's Theorem on the Moment Problem of Closed Semi-algebraic Sets, Proc. of the Amer. Math. Soc. 136 (2008), 529-537. MR 2358493 (2009a:44012)

[N2] T. Netzer: Stability of Quadratic Modules, Manuscripta Mathematica, 129(2), 251-271 (2009). MR 2505804

[Pl] D. Plaumann: Bounded Polynomials, Sums of Squares and the Moment Problem, Doctoral Thesis, University of Konstanz (2008).

[PoSc] V. Powers, C. Scheiderer: The Moment Problem for Non-compact Semialgebraic Sets, Adv. Geom. 1 (2001), 71-88. MR.1823953 (2002c:14086)

[Po] V. Powers: Positive Polynomials and the Moment Problem for Cylinders with Compact Cross-section, J. Pure Appl. Alg. 188 (2004), 217-226. MR2030815 (2004k:14107)

[PD] A. Prestel, C. N. Delzell: Positive Polynomials, Springer, Berlin (2001). MR1829790 (2002k:13044)

[Pu] M. Putinar: Positive Polynomials on Compact Semi-algebraic Sets, Indiana Univ. Math. J. 3 (1993), 969-984. MR.1254128 (95h:47014)

[Sf] H.H. Schaefer: Topological Vector Spaces, 2nd Edition, Springer, New York (1999). MR0342978 (49:7722)

[Sc1] C. Scheiderer: Sums of Squares of Regular Functions on Real Algebraic Varieties, Trans. Amer. Math. Soc. 352 (1999), 1039-1069. MR.1675230 (2000j:14090)

[Sc2] C. Scheiderer: Sums of Squares on Real Algebraic Curves, Math. Z. 245 (2003), 725-760. MR 2020709 (2004k:14103)

[Sc3] C. Scheiderer: Distinguished Representations of Non-negative Polynomials, J. Algebra 289 (2005), 558-573. MR2142385 (2006d:13025)

[Sc4] C. Scheiderer: Non-existence of Degree Bounds for Weighted Sums of Squares Representations, Journal of Complexity 21 (2005), 823-844. MR.2182447 (2006k:14117)

[S1] K. Schmüdgen: The K-moment Problem for Compact Semi-algebraic Sets, Math. Ann. 289 (1991), 203-206. MR.1092173 (92b:44011)

[S2] K. Schmüdgen: On the Moment Problem of Closed Semi-algebraic Sets, J. reine angew. Math. 558 (2003), 225-234. MR.1979186 (2004e:47019)

[Sw1] M. Schweighofer: Iterated Rings of Bounded Elements and Generalizations of Schmüdgen's Positivstellensatz, J. Reine Angew. Math. 554 (2003), 19-45. MR1952167 (2004b:13028)

[Sw2] M. Schweighofer: Optimization of Polynomials on Compact Semialgebraic Sets, SIAM J. Optim. 15 (2005), 805-825. MR2142861 (2006d:90136)

FAKUltät FÜr Mathematik Und Informatik, Universität LeipZig, PF 100920, 04009 LEIPZig, Germany

E-mail address: tim.netzer@math.uni-leipzig.de 